\title{
Autophagy promotes survival of retinal ganglion cells after optic nerve axotomy in mice
}

\author{
N Rodríguez-Muela', F Germain², G Mariño ${ }^{3}$, PS Fitze ${ }^{4,5}$ and P Boya ${ }^{\star, 1}$
}

\begin{abstract}
Autophagy is an essential recycling pathway implicated in neurodegeneration either as a pro-survival or a pro-death mechanism. Its role after axonal injury is still uncertain. Axotomy of the optic nerve is a classical model of neurodegeneration. It induces retinal ganglion cell death, a process also occurring in glaucoma and other optic neuropathies. We analyzed autophagy induction and cell survival following optic nerve transection (ONT) in mice. Our results demonstrate activation of autophagy shortly after axotomy with autophagosome formation, upregulation of the autophagy regulator Atg5 and apoptotic death of $50 \%$ of the retinal ganglion cells (RGCs) after 5 days. Genetic downregulation of autophagy using knockout mice for Atg4B (another regulator of autophagy) or with specific deletion of $A \operatorname{tg} 5$ in retinal ganglion cells, using the $A \operatorname{tg} 5^{\text {flox/flox }}$ mice reduces cell survival after ONT, whereas pharmacological induction of autophagy in vivo increases the number of surviving cells. In conclusion, our data support that autophagy has a cytoprotective role in RGCs after traumatic injury and may provide a new therapeutic strategy to ameliorate retinal diseases.
\end{abstract}

Cell Death and Differentiation (2012) 19, 162-169; doi:10.1038/cdd.2011.88; published online 24 June 2011

Retinal ganglion cells (RGCs) are the only projecting neurons of the retina. Their axons form the optic nerve and transmit visual information to the brain. RGCs undergo apoptotic cell death in a stereotyped manner during development and in response to injury, in glaucoma and other optic neuropathies. ${ }^{1}$ Degeneration of RGCs is often modeled by optic nerve transection (ONT), which leads to the death of these central nervous system neurons. ${ }^{2}$ The mechanisms of RGC death are still a matter of intense investigation, and several factors including growth factor deprivation and oxidative stress have been proposed to participate in RGC degeneration in glaucoma and after ONT. ${ }^{1,3}$

Autophagy is an intracellular catabolic pathway, which degrades cell components, toxic aggregates and damaged organelles and recycles them as basic building blocks in order to maintain cellular homeostasis. ${ }^{4}$ Autophagy begins with the formation of a double membrane, sequestering parts of the cytosol and finally closing to form an autophagosome. This autophagosome subsequently fuses with lysosomes, thus, enabling degradation of the engulfed material. ${ }^{4}$ Autophagy represents a cytoprotective response in many cell types ${ }^{5}$ and its deregulation is implicated in many pathological conditions, including cancer, infectious diseases and neurodegeneration. ${ }^{6}$

The role of autophagy in neuronal physiology is still far from being completely understood. ${ }^{7,8}$ On one hand, autophagy is essential in preventing spontaneous neurodegeneration in mice, as deletion of the autophagy regulators Atg5, Atg7 and FIP200 in neuronal precursors induces cell death, accumulation of damaged ubiquitinated proteins and premature lethality. ${ }^{9-11}$ Similarly, upregulation of autophagy decreases the accumulation of protein aggregates in several neurodegenerative proteinopathies. ${ }^{12,13}$ Conversely autophagy triggers neuronal death under several circumstances such as after hypoxic/ischemic brain injury. ${ }^{14,15}$

The retina is an accessible region of the central nervous system in which autophagy was shown to be activated in a rat model of ONT, as well as in a RGC line after serum starvation. ${ }^{16}$ Few studies have addressed the role of autophagy in this organ in vivo, reporting opposite results as both cytoprotective and procell death effects have been described in photoreceptors in fly ${ }^{17}$ and mouse models, ${ }^{18}$ whereas detrimental effects of autophagy have also been described after optic nerve damage. ${ }^{19,20}$ The goal of our study was to understand the function of autophagy following ONT in vivo. Here we demonstrate that autophagy is activated shortly after optic nerve axotomy in mice and has a cytoprotective role. By using a recently described knockout mouse model of autophagy impairment we demonstrate that genetic downregulation of autophagy increases RGC death, whereas pharmacological upregulation of autophagy reduces $\mathrm{RGC}$ loss in vivo. Thus, autophagy modulation may represent a new therapeutic target to ameliorate RGC degeneration in optic neuropathies.

\footnotetext{
${ }^{1}$ Department of Cellular and Molecular Medicine, 3D Lab, Development, Differentiation and Degeneration, Centro de Investigaciones Biológicas, CSIC, Ramiro de Maeztu 9, 28040 Madrid, Spain; ${ }^{2}$ Department of Physiology, Universidad de Alcalá, 28871 Alcalá de Henares, Spain; ${ }^{3}$ Department de Bioquímica y Biología Molecular, Instituto Universitario de Oncología, Universidad de Oviedo, 33006 Oviedo, Spain; ${ }^{4}$ Department of Biodiversity and Evolutionary Biology, Museo Nacional de Ciencias Naturales CSIC, Madrid 28006, Spain and ${ }^{5}$ Department of Ecology and Evolution, Biophore, University of Lausanne, 1015 Lausanne, Switzerland

${ }^{*}$ Corresponding author: P Boya, Department of Cellular and Molecular Medicine, 3D Lab, Development, Differentiation and Degeneration, Centro de Investigaciones Biológicas, CSIC, Ramiro de Maeztu 9, E-28040 Madrid, Spain. Tel: + 34918373112 (ext 4369); Fax: + 34915360432 ; E-mail: pboya@cib.csic.es Keywords: apoptosis; autophagy; neurodegeneration; retina; retinal ganglion cells

Abbreviations: 3-MA, 3-methyladenine; AAV, adeno-associated virus; AP, autophagosome; DAPI, 4',6-diamidino-2-phenylindole; DCF, dichloro-fluorescein; $\mathrm{DHE}$, di-hydroethidium; DiOC6(3), 3,3'-dihexyloxacarbocyanine iodide; DTMR, dextran tetramethylrhodamine; GCL, ganglion cell layer; GFP, green fluorescent protein; i.p., intraperitoneal; LC3, microtubule-associated protein 1 light chain 3; ONT, optic nerve transection; PD, Parkinson's disease; PI, propidium iodide; PQ, paraquat; RGC, retinal ganglion cell; RGC-5, retinal ganglion cell clone 5; ROS, reactive oxygen species; S.E.M., standard error of mean

Received 17.1.11; revised 15.4.11; accepted 27.4.11; Edited by M Piacentini; published online 24.6.11
} 


\section{Results}

RGC death and autophagy activation after optic nerve axotomy in mice. In order to characterize the precise dynamics of RGC death after ONT, optic nerves from adult mice were unilaterally sectioned and analyzed at different time points after the injury. RGCs were immunostained against Brn-3a, a specific transcription factor of RGCs, and counted in the ganglion cell layer (GCL), which is composed both by RGCs and displaced amacrine cells, a cell type unaffected by axotomy as their prolongations do not form part of the optic nerve. A significant decrease in the number of RGCs was observed starting at 5 days after axotomy by Brn-3a immunostaining (Figures 1a and $c$ ), which was confirmed by retrograde labeling of RGCs with dextran tetramethylrhodamine (DTMR) (Figures $1 b$ and $d$ ) and by immunoblotting of whole retinal extracts against neuronal markers (Supplementary Figure 1A). In agreement, a decrease in the total number of cells in the GCL stained with DAPI was observed (Supplementary Figure 1B). Dying RGCs displayed pyknotic nuclei (Figures $2 a$ and b), intranucleosomal DNA fragmentation (Supplementary Figure 1C) and activated caspase-3 (Figures 2c and d). Together these data demonstrate that optic nerve axotomy in mice induces apoptotic cell death of RGCs, peaking at 5 days after the lesion.

Autophagy induction was studied in parallel by performing ONT in GFP-LC3 mice. This mouse constitutes a reliable model to monitor autophagic activity in vivo by visualizing $A P$ formation with the autophagosomal-associated protein LC3 coupled to GFP. ${ }^{21}$ In control retinas, the RGCs only manifest a diffuse cytosolic GFP-LC3 pattern (Figures $3 a$ and b, left panels) in agreement with previous studies in which neurons show low levels of autophagy under basal conditions. ${ }^{9}$ However, axotomized retinas showed a significant increase in autophagosome-positive cells in comparison with control retinas, starting 3 days after ONT (Figures $3 a-c)$ ). Autophagosomes were visualized as green dots in the cytosol (Figure $3 \mathrm{~b}$ )
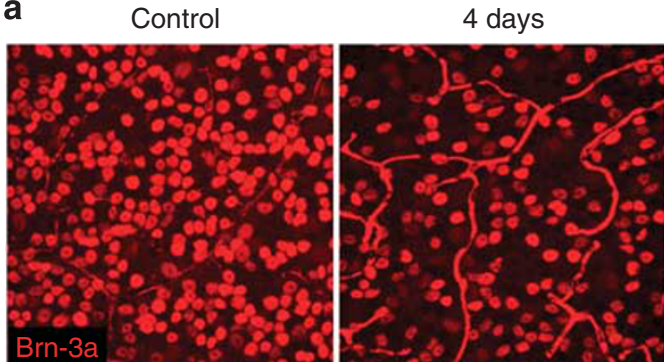

4 days
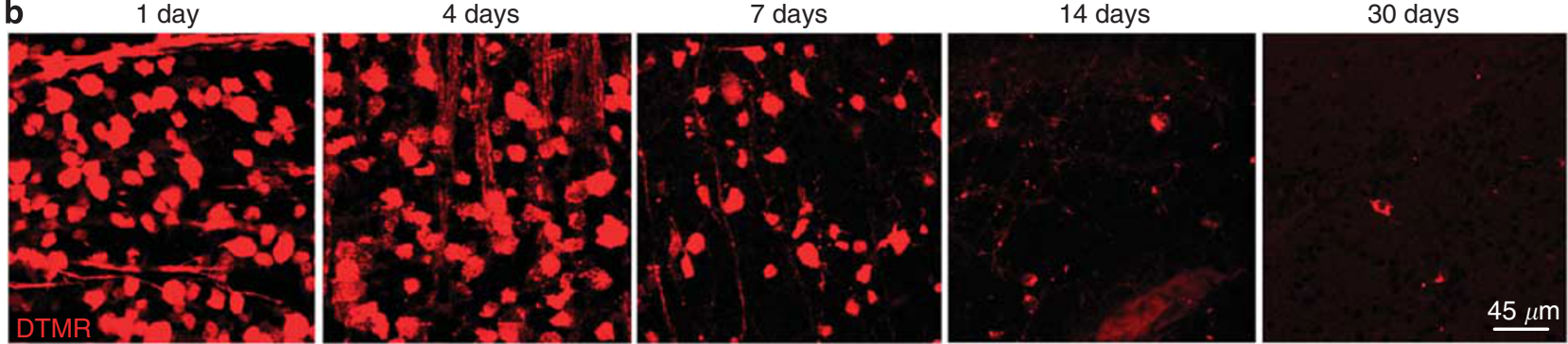

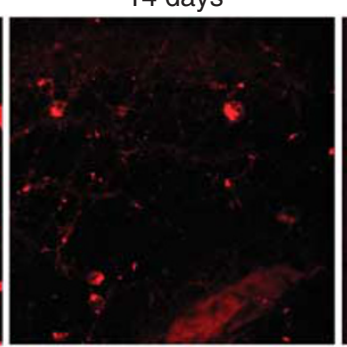

10 days

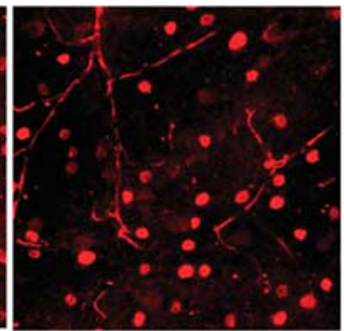

14 days
Axotomy

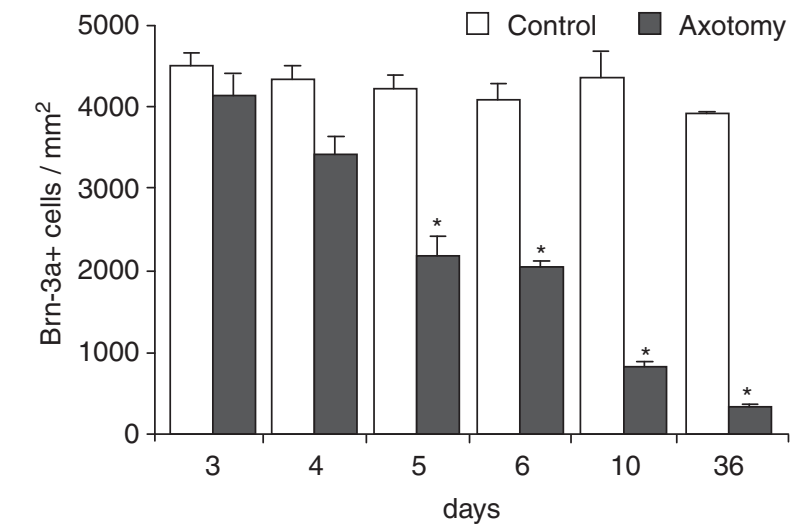

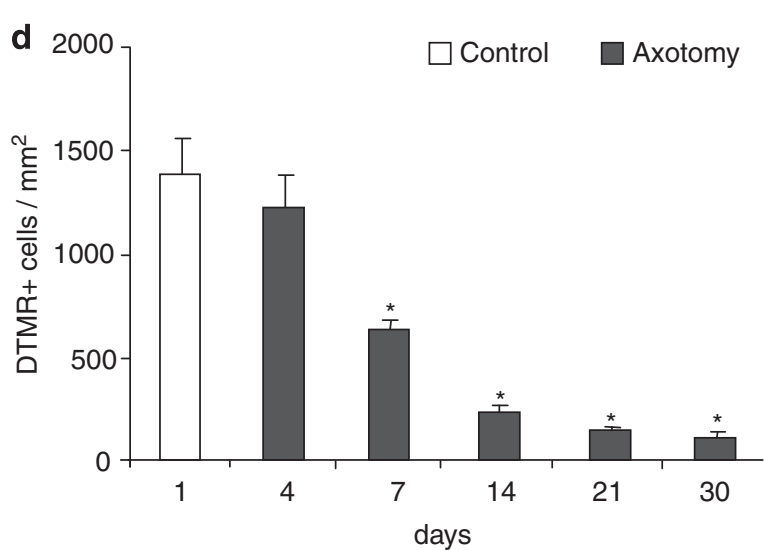

$\square$ Control

Figure 1 Retinal ganglion cell loss after ONT. Optic nerves from adult mice were unilaterally sectioned and the animals were killed at different time points. Right (control) and left (axotomy) retinas were then isolated, flat-mounted and fixed. (a and c) Immunostaining and quantification of the number of Brn-3a-positive cells in control and axotomized retinas. (b-d) DTMR retrograde labeling of RGCs and its quantification from one day (control) up to 30 days of axotomy 
a

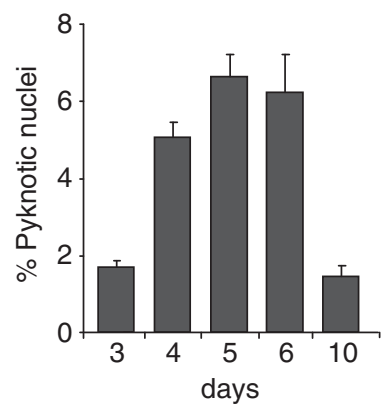

b

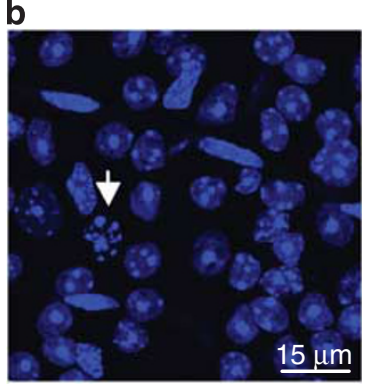

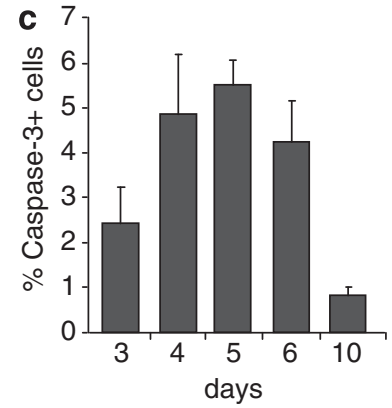

d Control

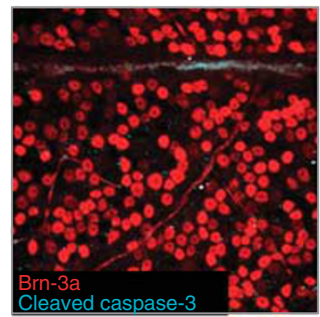

4 days

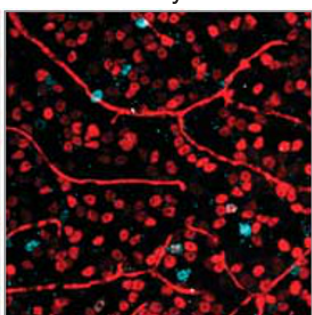

6 days

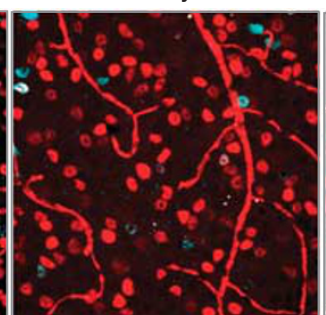

10 days

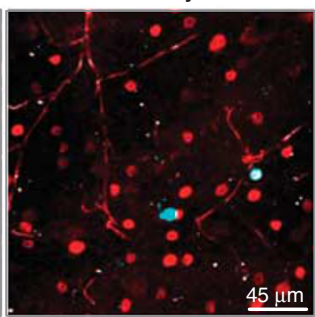

Figure 2 Kinetics of cell death after ONT. (a) Quantification of the percentage of pyknotic nuclei stained with DAPI related to the total number of cells in the ganglion cell layer (GCL). (b) Pyknotic nuclei stained with DAPI (arrow) in the GCL for 6 days after axotomy. (c) Quantification of the percentage of activated caspase-3-positive cells and (d) immunostaining against Brn-3a and cleaved caspase-3 in control and axotomized retinas at different time points after axotomy

and specifically induced in RGCs as this green dotted-pattern was only observed in the Brn-3a-positive cells (Supplementary Figure $2 \mathrm{~A}$ ) and no changes were observed in the other layers of retinal cells. An increase in LC3-II, the lipidated autophagosome-associated form of LC3, and Beclin 1 can also be observed at 4 days after the injury (Supplementary Figure 2B). These differences were less pronounced as immunoblot was performed in total retinal extracts and RGCs comprise $<5 \%$ of total cells in the adult mouse retina. Consistently, autophagy was upregulated at the transcriptional level as Atg5 - an essential component for autophagosome formation - mRNA expression was induced (Figure 3d). In addition, ultrastructural analyses of axotomized retinas showed that RGCs presented pyknotic nuclei, autophagosomes and dilated endoplasmic reticulum (Figure 3e). Moreover, swollen mitochondria with altered cristae and weak electrodense lumen, which were frequently engulfed by autophagosomes, were often observed (Figure 3e). Together our data show that autophagy upregulation occurs 3 days after ONT and before cell death peak.

Pharmacological induction of autophagy reduces cell death and oxidative damage. Oxidative damage has been implicated in RGC death after optic nerve axotomy and during glaucomatous neuropathy. ${ }^{3}$ As expected, axotomy induced an increase in reactive oxygen species (ROS) levels in RGCs determined by di-hydroethidium (DHE) staining in vivo (Figure 4a), a probe that particularly detects superoxide anion levels, one of the two ROS species considered as the major autophagy inductors. ${ }^{22}$ When we challenged RGC clone 5 (RGC-5) retinal cells with the ROSinducing agent paraquat (PQ), we observed loss of mitochondrial membrane potential, enhanced ROS production and an increase in cell death (Figures $4 b-d$ and Supplementary Figures $3 \mathrm{~A}$ and $\mathrm{B}$ ). In order to study whether the autophagy upregulation observed in vivo after ONT contributes to cell death or by contrast, represents a cytoprotective response, we modulated autophagy in vitro in RGC-5 cells. Rapamycin treatment induced autophagy activation as determined by increased levels of LC3-II and a reduction of the LC3-binding protein and autophagy substrate p62 (Figure 4e) and protected from cell death induced by $P Q$ toxicity (Figure $4 d$ ). Treatment with $P Q$ alone induced an increase in p62 levels, according to previous studies in which an upregulation of this protein was observed under an oxidative stress situation. ${ }^{23}$ Rapamycin also preserved mitochondrial membrane potential and decreased intracellular ROS production (Figures $4 b$ and $c$ ). Conversely, pharmacological autophagy inhibition by 3-methyladenine (3-MA) reduced cell viability, decreased mitochondrial membrane potential and increased ROS production in untreated cells (Figures $4 b-d$ ). In PQ-treated cells, 3-MA also increased cell death at earlier time points (Supplementary Figure 3B). Altogether these data confirm that ROS are negatively correlated with mitochondrial membrane potential and positively with the incidence of cell death. Further, autophagy induction protects RGC-5 cells from mitochondrial damage and cell death, whereas autophagy inhibition promotes ROS production and cell death.

Autophagy induction increases RGC survival, whereas its genetic downregulation reduces cell viability in vivo. To test whether an increase in autophagy could protect retinal cells from axotomy-induced degeneration, axotomized WT mice were treated with the autophagyinductor rapamycin. We observed a significant increase 
a

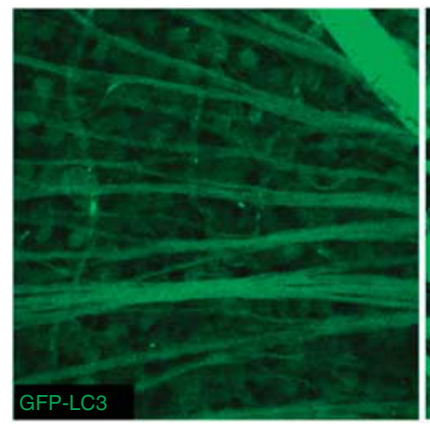

b
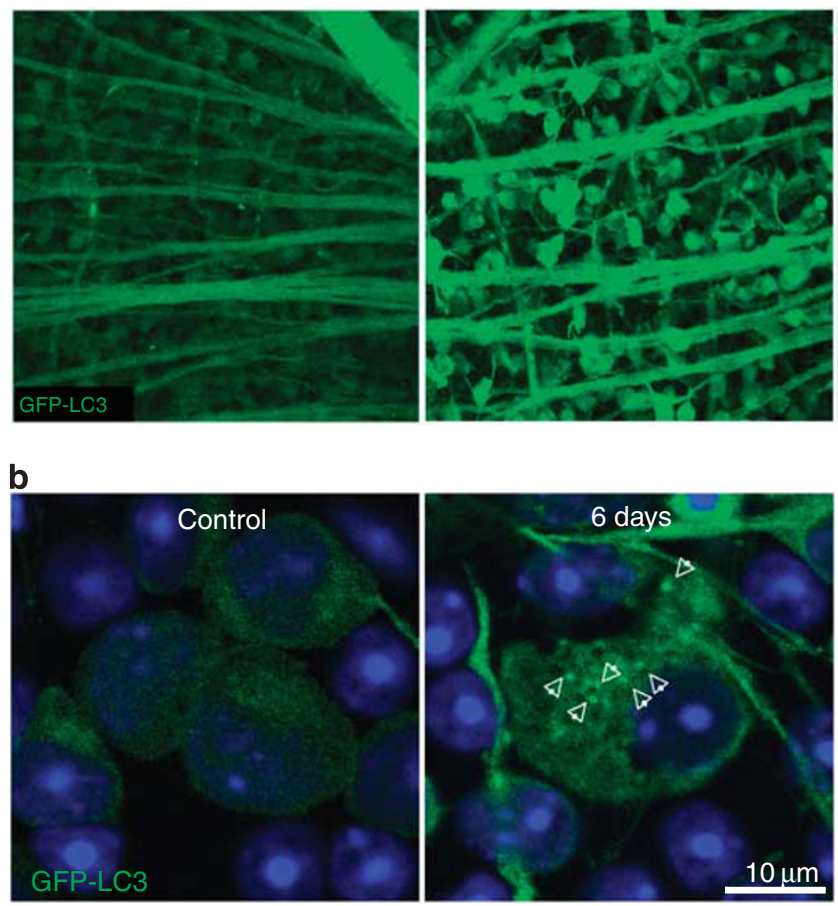

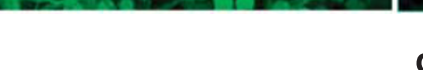

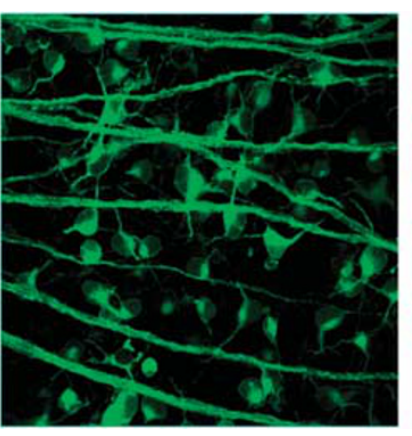

c

$\square$ Control $\square$ Axotomy

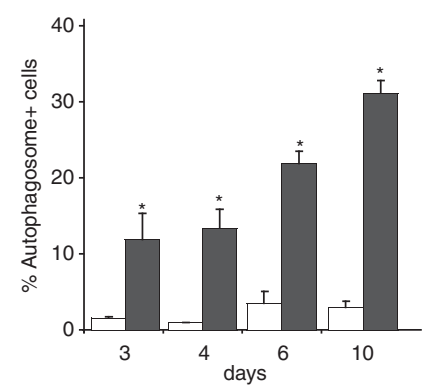

10 days

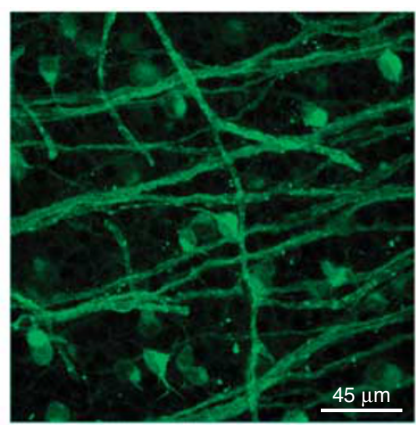

d $\square$ Control $\square$ Axotomy

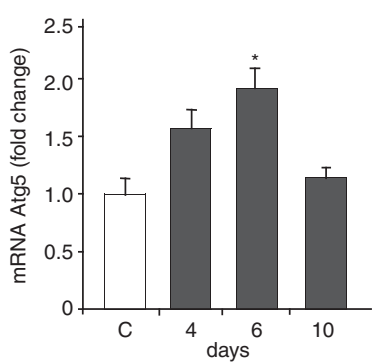

e
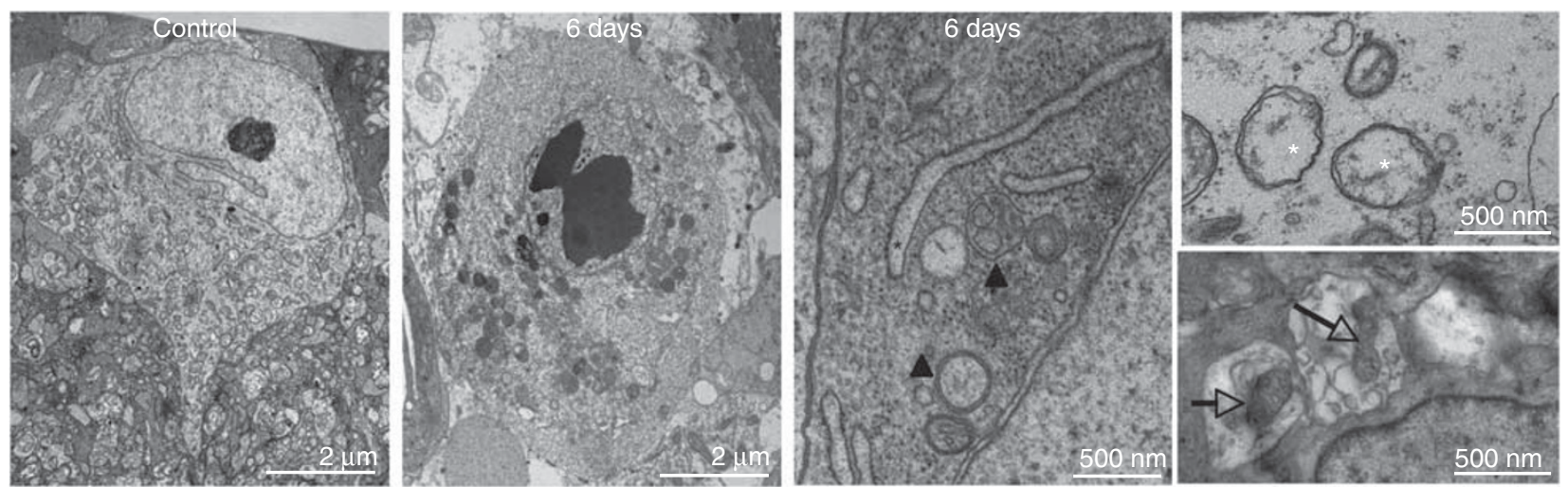

Figure 3 Autophagy is activated in RGCs shortly after ONT. (a) GFP-LC3 fluorescence of the GCL in flat-mounted retinas from control and axotomized mice. (b) Magnification of RGCs in a control retina and 6 days after ONT, a $0.5 \mu \mathrm{m}$ confocal plane is shown. Cells from control retinas display a diffuse and homogeneous green fluorescence in the cytosol, whereas the axotomized RGCs in the right panel showt visible GFP-LC3 dots in the cytosol identified as autophagosomes and pointed with arrowheads. (c) Quantification of the percentage of cells with GFP-LC3 positive APs in the cytosol versus total number of cells in the GCL. (d) Analysis of Atg5 mRNA levels by qPCR in control and axotomized retinas at different time points after the injury. (e) Electron microscopy analysis of representative RGCs from control and 6 days-injured retinas. Panels on the right show a magnification of a degenerating RGC after the lesion. Note the swollen endoplasmic reticulum (black asterisk), damaged mitochondria (white asterisks), autophagosomes (black arrow head) and mitochondria inside autophagosomes (empty arrows)

(40\%) in the number of surviving RGCs after 10 days as compared with the vehicle-treated animals (Figure 5a). Interestingly, this cytoprotective effect correlated with an increase in the ratio LC3-II/LC3-I in whole retinas from mice treated with rapamycin during 10 days (Figure $5 \mathrm{~b}$ and Supplementary Figure 4A). Note that this increase is rather modest as the western blot is performed in whole retinal extracts and RGCs, the only cell type affected by the injury and in which autophagy is activated, represent $<5 \%$ of the total cells of the adult mouse retina. Together these results demonstrate that autophagy induction is beneficial and protective after injury in vivo in agreement with our in vitro results.

To further confirm these data, we took advantage of the recently described $A \operatorname{tg} 4 B^{-1-}$ mice. ${ }^{24}$ These mice are viable and fertile, they display reduced autophagy levels in many tissues including the brain, and increased levels of ubiquitinated proteins and the autophagy substrate p62. ${ }^{24}$ As

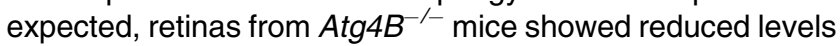
of LC3 lipidation and increased levels of p62, indicating that basal autophagy is reduced in the retinas (Figure $5 \mathrm{~d}$ and Supplementary Figures $4 B$ and C). Atg4B deletion did not 
a
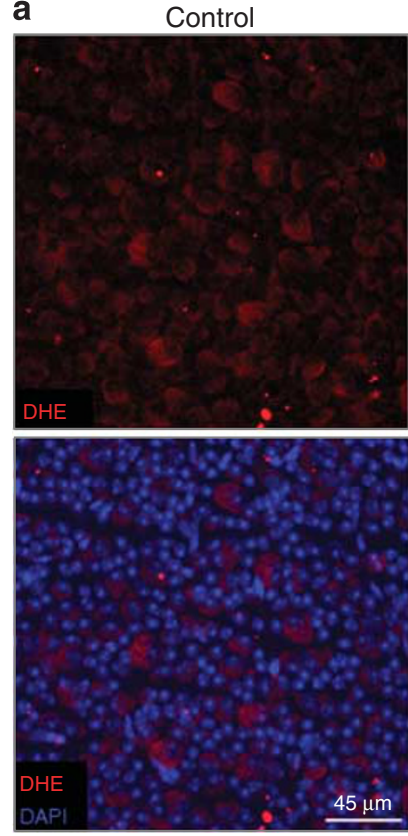

6 days
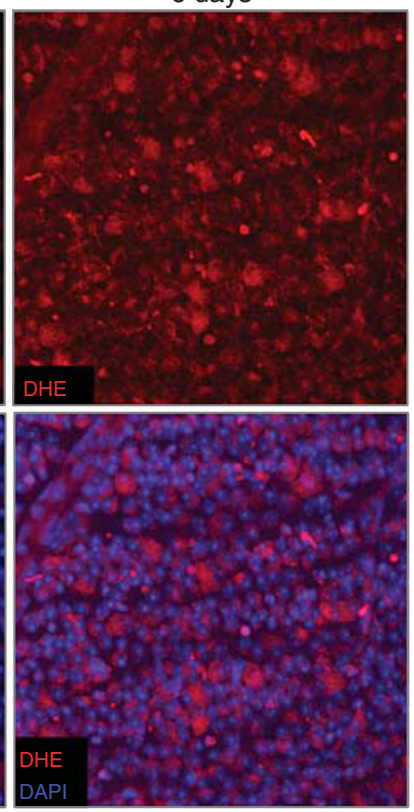

b
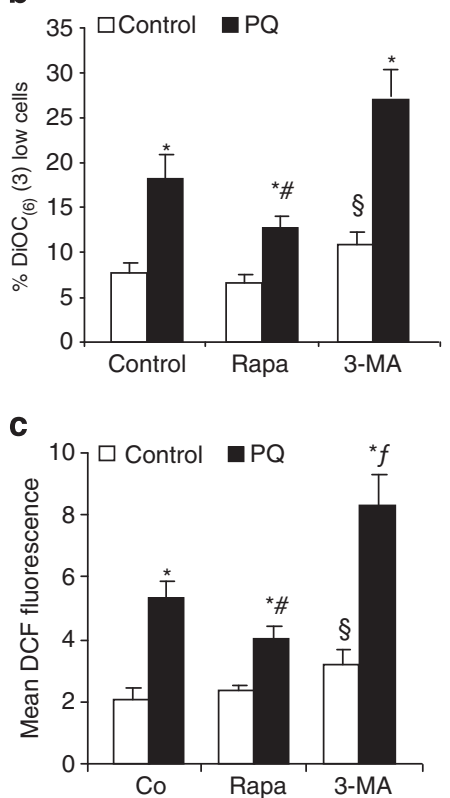
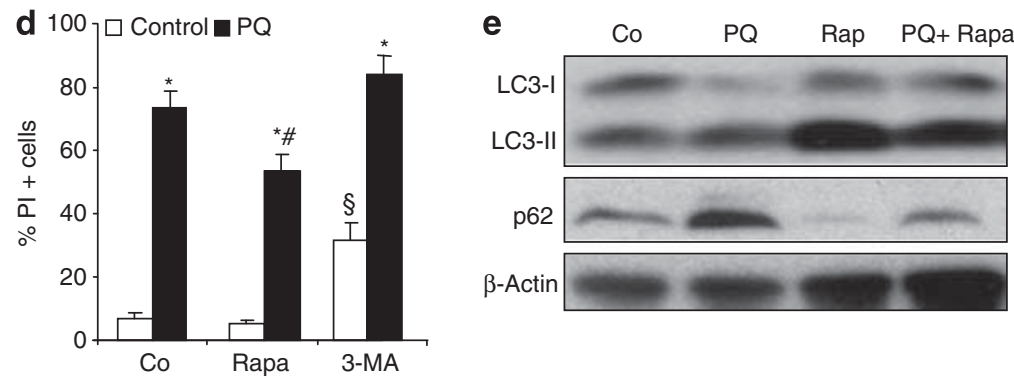

Figure 4 ROS production in vivo and in vitro and autophagy modulation in vitro. (a) In vivo ROS staining in the GCL of control and 6 days-axotomized retinas after injecting the animals with DHE for $18 \mathrm{~h}$. (b-d) FACS analysis of RGC-5 cells treated with paraquat (PQ) alone or combined with rapamycin or 3-MA to determine (b) mitochondrial membrane potential with $\mathrm{DiOC}_{(6)}(3)$ at $24 \mathrm{~h}$, (c) ROS production with DCF at $48 \mathrm{~h}$ after treatment and (d) cell viability with $\mathrm{PI}$ staining at $48 \mathrm{~h}$ and. ${ }^{*} P<0.05 \mathrm{Control}$ versus $\mathrm{PQ}$, ${ }^{\#} P<0.05 \mathrm{PQ}$ versus Rapa $+\mathrm{PQ},{ }^{8} P<0.05$ Control versus $3-\mathrm{MA},{ }^{f} \mathrm{P}<0.05 \mathrm{PQ}$ versus $\mathrm{PQ}+3$-MA. (e) Western blot analysis of RGC-5 cells treated with rapamycin and subjected to $P Q$ toxicity during $24 \mathrm{~h}$

alter retinal function as determined by electroretinogram in adult mice, and intact retinas contained equal number of RGCs compared with WT animals (data not shown), indicating that autophagy downregulation in these mutant mice does not induce major alterations in visual function and RGC generation. To test whether their reduced basal autophagy could influence RGCs survival after axotomy, we performed

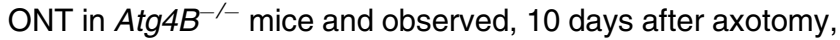
a reduced number of surviving RGCs ( $28 \%$ less) as compared with WT mice (Figure 5c). These data support our hypothesis that autophagy increases RGCs survival after injury.

To discard that these effects on RGC death resulted from indirect autophagy modulation in neighboring cells, we exclusively eliminated Atg5 in RGCs. This was achieved by intravitreal injection of the adenovirus AAV-Cre-GFP serotype2, which specifically transduces RGCs, into $\operatorname{Atg} 5^{\text {flox/flox }}$ mice. ${ }^{9}$ Consistent with our observations on $\operatorname{Atg}^{4} B^{-1-}$ mice, AAV-Cre-GFP injected retinas displayed reduced numbers of surviving RGCs (33\%) after axotomy as compared with those injected with the reporter AAV-GFP (Figure 5e). Injection of AAV-Cre-GFP increased the accumulation of p62 and ubiquitin-positive proteins (Figure $5 f$ ), ${ }^{9}$ although a clear reduction in LC3 lipidation in retinal protein extracts was not observed, likely because of the fact that RGCs represent $<5 \%$ of total cells in the retina ${ }^{1}$ and also because adenoviral infection does not transduce all cells (Supplementary Figures 4D and E). Together our results demonstrate that genetic downregulation of autophagy by two distinct approaches reduces RGC survival after optic nerve injury in vivo.

\section{Discussion}

In this work we have addressed the role of autophagy in retinal neurodegeneration by combining in vitro and in vivo, pharmacological and genetic approaches. First, by using an acute experimental model of ONT in mouse that induces RGC death in combination with a transgenic mouse model that allows monitoring autophagy in vivo, we have found that autophagy is rapidly induced after ONT, also at the transcriptional level, before RGC cell death. Further, we have demonstrated that the observed autophagy upregulation after axotomy constitutes a cytoprotective response. Pharmacological 
a
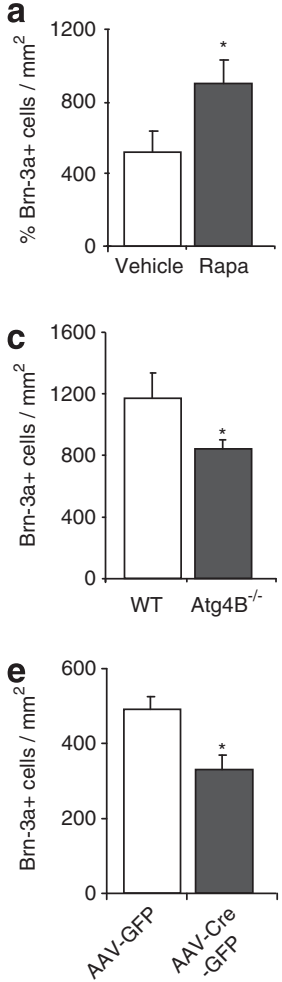
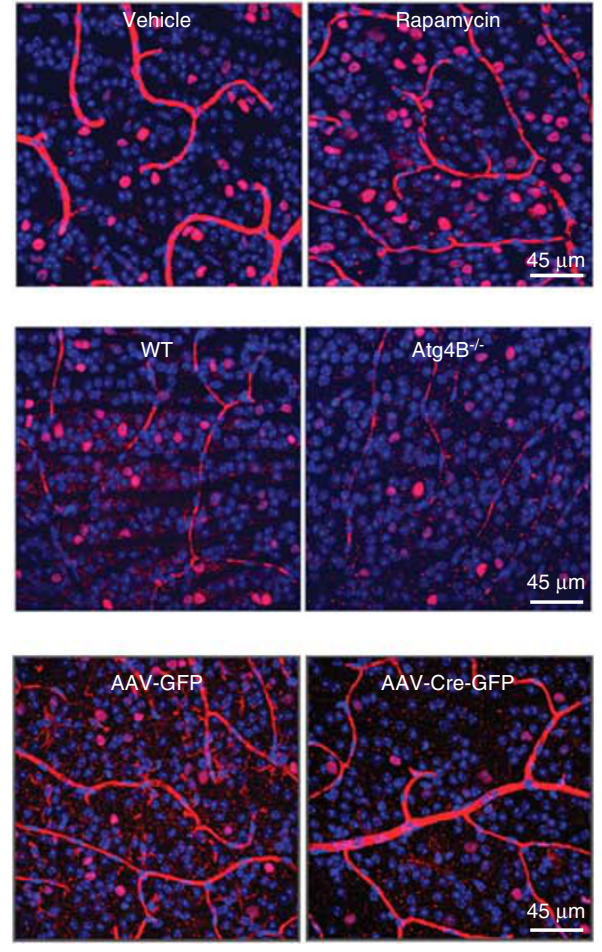

b
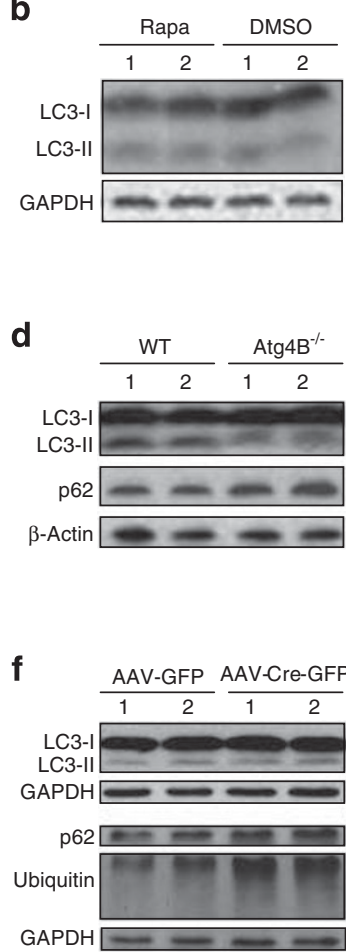

Figure 5 Autophagy protects after ONT in vivo. (a) Representative image showing Brn-3a immunostaining of vehicle and rapamycin-treated animals and quantification of the percentage of surviving RGCs 10 days after axotomy. (b) LC3 immunoblot of retinas from mice injected with vehicle or rapamycin at 10 days after the injury. (c) Image of RGCs stained with Brn-3a from WT and Atg4B ${ }^{-1}$ mice at 10 days after axotomy and quantification of the percentage of RGCs in the GCL. (d) Immunoblot analysis of untreated WT and $A \operatorname{Atg} 4 B^{-1-}$ retinas. (e) Representative image of the GCL stained for Brn-3a and its quantification at 10 days after ONT in Atg $5^{\text {floxflox }}$ mice injected with AAVGFP or AAV-Cre-GFP vectors. (f) Immunoblot analysis of uninjured retinas from mice injected with AAV-GFP or AAV-Cre-GFP

upregulation of autophagy reduces axotomy-induced RGC loss, whereas mice with a reduced autophagy activity because of a deletion in the autophagy $A \operatorname{tg} 4 B$ regulator display a reduced number of surviving RGCs after injury. Moreover, specific deletion of the autophagy essential gene Atg5 in retinal neurons before injury also results in a decrease in the number of surviving cells. Altogether these data show for the first time the cytoprotective role of autophagy in cell soma after axonal insult.

The clasical model of ONT has been mostly performed in rats and rabbits, because open transection in mice is by far more complicated and difficult to carry out. ${ }^{1}$ This is a widely used model in glaucoma research as the only spontaneous type of this disorder with similar symptoms to the human pathology, the DBA/2J mouse, has a great variability and asymmetry in developing the disease, thus, large cohorts of animals must be used to obtain significant results. ${ }^{25,26}$ Some studies have determined the decrease in the number of RGCs after ONT or crushed mostly by retrograde labeling, but a detailed analysis of the kinetics of apoptotic cell death after ONT had not been previously performed in mice. Here, to finely characterize the kinetics of the process, we have performed a detailed time course by using several and complementary techniques: counts of pyknotic nuclei stained with DAPI, number of cells with activated caspase-3 by confocal microscopy and DNA fragmentation by ELISA. All these techniques provided congruent results, with a cell death peak around day 5 after injury. Importantly, cell counting by confocal microscopy in the GCL was by far the most sensitive technique as it only takes into account changes the GCL in contrast to other methods as western blot or ELISA that are performed in whole retinal extracts.

Autophagy dysfunction has been directly associated to a growing number of neurodegenerative disorders. ${ }^{8}$ We have recently found impaired autophagy in a mouse model of Parkinson disease (PD) and an accumulation of the autophagosome-associated LC3-II in PD patients. ${ }^{27}$ Importantly, rapamycin treatment prevented dopaminergic neurodegeneration in parkinsonian mice, ${ }^{27}$ confirming previous evidences that autophagy induction is beneficial in models of neurodegeneration, which course with protein aggregates in mouse ${ }^{12,13,28,29}$ and in fly models of retinal degeneration. ${ }^{17}$ In our hands, rapamycin treatment protects retinal neurons after ONT (40\% increase) in mice. Thus, our results extend to neuronal pathologies not associated with protein accumulation the spectrum of diseases in which autophagy has a cytoprotective role.

To finally demonstrate the beneficial effect of autophagy in our system autophagy-deficient animals were subjected to axonal injury, a strategy that has never been used in mice. First, we use the recently described $A \operatorname{tg} 4 B^{-1-}$ mice that have reduced levels of basal autophagy but are able to induce autophagic flux under several circumstances. ${ }^{24}$ The number of surviving RGCs after axotomy is lower in $\mathrm{Atg}^{4} \mathrm{~B}^{-/-}$mice compared with control mice, indicating that proper autophagy levels are needed to protect neurons after injury. The same 
result was obtained when Atg5 was specifically deleted in RGCs cells using the cre-lox strategy in the Atg $5^{\text {flox/flox }}$ mice. These data imply that autophagy is essential for RGC survival after ONT in adult individuals, precluding the possibility that the effects observed in $A \operatorname{tg} 4 B^{-/-}$mice were because of putative developmental defects caused by their decreased basal autophagic activity.

On one hand, we have found that there is an increase in the levels of ROS in the RGCs after the axonal injury, and by analyzing the ultrastructure of these degenerating neurons we have detected damaged mitochondria, some of them inside APs. On the other hand, our results demonstrate that the upregulation of autophagy activity leads to an extended neuronal life after a traumatic brain injury, hence, we postulate that autophagy might be involved in removing damaged mitochondria, which will in turn reduce the levels of ROS species and oxidized proteins. ${ }^{22}$ Our in vitro results indeed corroborate this theory as we demonstrate that rapamycin, through autophagy induction, protects RGCs in response to oxidative damage, whereas autophagy inhibition has the opposite effect. Importantly, the retina is one of the most commonly affected tissues in mitochondrial diseases, ${ }^{30}$ and essential proteins for mitophagy ${ }^{31}$ have a reduced expression in glaucomatous mice. ${ }^{32}$ Autophagy may also be involved in additional cytoprotective functions by removing axonal debris or remodeling cytoskeleton, ${ }^{33}$ and as a pro-survival response after growth factor deprivation that results from RGC disconnection from their targets and through provision of ATP to the retina. ${ }^{34}$

Altogether these data show for the first time the cytoprotective role of autophagy in cell soma after axonal insult and may provide the basis for new regeneration therapies, in which prolongation of neuronal soma survival is crucial for axonal maintenance and regeneration. It is important to remark the great degree of neuroprotection achieved in such a drastic model. This fact indicates that autophagy upregulation may be highly relevant in milder situations of axonal damage such as glaucoma and other optic neuropathies and points out autophagy modulation as a promising clinical target to ameliorate retinal degenerative disorders.

\footnotetext{
Materials and Methods

Animal procedures. Experiments were approved by the local ethics committee for animal experimentation, and carried out in accordance with the EU guidelines and the ARVO Statement for the Use of Animals in Ophthalmic and Vision Research. GFP-LC3 transgenic mice ${ }^{21}$ and $A \operatorname{tg} 5^{\text {floxfloxy }}$ were provided by Noboru Mizushima. Atg4B ${ }^{-1-}$ mice were provided by Carlos López-Otín. ${ }^{24}$ In order to perform ONT, animals were anaesthetized with $80 \mathrm{mg} / \mathrm{kg}$ intraperitoneal (i.p.) injection of ketamine chlorhydrate solution (Merial, Barcelona, Spain) and $10 \mathrm{mg} / \mathrm{kg}$ $2 \%$ xylacine chlorhydrate (Bayer, Barcelona, Spain). Left optic nerves were intraorbitally sectioned at $1 \mathrm{~mm}$ from the eyeball, and right eyes were used as control. Care was taken not to injure blood vessels and this was confirmed by fundus ophthalmoscopy. After surgery, antibiotic solution (Terra-Cortril; Pfizer, Girona, Spain) and analgesic (Buprenorphine; Bedford Laboratories, Bedford, OH, USA) were administered. Retrograde labeling was performed by placing a piece of spongostan absorbed with $0.1 \%$ fluorescent tracer DTMR (Molecular Probes, Paisley, UK) on the optic nerve stump after surgery. ROS were measured in RGCs in vivo after the axotomy, $18 \mathrm{~h}$ before killing, by an i.p. injection of DHE (Invitrogen, Paisley, UK), which specifically measures $\mathrm{O}_{2}^{-}$levels, at $25 \mathrm{mg} / \mathrm{kg}$, dissolved in DMSO and diluted right before administration in $5 \%$ Tween $80,5 \%$ polyethylene glycol 400 and $0.9 \% \mathrm{NaCl}$. For intraocular injection, Atg $5^{\text {flox }}$ flox mice were anesthetized and the left eyes injected with a 35-gauge needle in the lateral zone
}

$1 \mathrm{~mm}$ behind sclero-corneal limbus with $1 \mu$ of the adenovirus AAV2-GFP or AAV2Cre-GFP (Vector Biolabs, Philadelphia, PA, USA). Rapamycin (Euromedex, Souffelweyersheim, France), dissolved in DMSO, was diluted right before administration in $5 \%$ Tween $80,5 \%$ polyethylene glycol 400 and $0.9 \% \mathrm{NaCl}$, and injected i.p. at $7.5 \mathrm{mg} / \mathrm{kg}$ at 2 days before the injury, and afterward daily until killing with an overdose of sodium pentobarbital.

The number of animals used per experiment was the following: Figures $1 \mathrm{a}$ and $\mathrm{c}$ : $n=23 ; \mathrm{b}, \mathrm{d}: n=18$. Figures 2a-d: $n=23$. Figures 3a-c: $n=16 ; 3 \mathrm{~d}: n=25$; 3e: $n=12$. Figure 4a: $n=6$. Figure $5 \mathrm{a}: n=14 ; 5 \mathrm{~b}: n=10 ; 5 \mathrm{c}: n=18 ; 5 \mathrm{~d}: n=12$; 5e: $n=10,5 f: n=8$. Supplementary Figure $1 \mathrm{~A}: n=9$; Supplementary figure B: $n=23$; Supplementary Figure 1C: $n=42$. Supplementary Figure $2 A: n=16$; Supplementary Figure 2B: $n=9$. Supplementary Figure $4 A: n=10$; Supplementary Figures $4 \mathrm{~B}$ and C: $n=12$; Supplementary Figures $4 \mathrm{D}$ and E: $n=10$.

Immunohistofluorescence and cell death ELISA. Retina isolation and immunohistofluorescence was performed as previously described ${ }^{34}$ with Brn-3a (Millipore, Billerica, MA, USA), cleaved caspase 3 (Cell Signaling, Boston, MA, USA) and alexa-conjugated secondary antibodies (Invitrogen). GCL was analyzed on a confocal microscope (TCS SP2; Leica Microsystems, Barcelona, Spain). Cell counting was performed by using the Image $\mathrm{J}$ software (NIH, Bethesda, MD, USA) on maximal confocal projections of eight fields per retina acquired with the $\times 63$ objective. DNA fragmentation was determined using a Cell Death ELISA kit (Roche, Basel, Switzerland) that quantifies the histone-associated DNA fragments (mono- and oligonucleosomes) produced during apoptosis. Control and axotomized retinas were individually homogenized in $400 \mu \mathrm{l}$ incubation buffer and cleared by centrifugation at $12000 \times g$ for $15 \mathrm{~min} ; 2.5 \mu \mathrm{l}$ of supernatant in a final volume of $25 \mu \mathrm{l}$ was added to the ELISA plate.

Quantitative RT-PCR. RNA isolation and RT were performed for individual retinas as previously described. ${ }^{34} \mathrm{Q}-\mathrm{PCR}$ was performed with $100 \mathrm{ng}$ CDNA with a TaqMan Universal PCR Master mix using probes from the Universal ProbeLibrary Set in a 7900 HT-Fast Real-Time PCR System (Roche Applied Biosystems, Pleasanton, CA, USA). Each value was adjusted by using $18 \mathrm{~S}$ RNA levels as a reference. Primer sequences were atg5F 5'-GAATATTTTGAATCTCCCGTTCC-3', atg5R 5'-GGCCACAATGACATAATCCA-3', 18sF 5'-TGCGAGTACTCAACACCAA CA-3' and 18sR $5^{\prime}$-TTCCTCAACACCACATGAGC- $3^{\prime}$.

Western blot. Western blot analysis was performed as previously described, ${ }^{5}$ with $\beta$-III tubulin (Covance, Princeton, NJ, USA), LC3 (MBL International, Woburn, MA, USA), p62 (Enzo Life Science AG, Lausen, Switzerland), Ubiquitin (Santa Cruz Biotechnology, Heidelberg, Germany), $\beta$-actin (Sigma, Madrid, Spain) and GAPDH (Abcam, Cambridge, UK) antibodies. Densitometric analysis was performed with Quantity One software (Bio-Rad, Alcobendas, Spain).

Transmission electron microscopy. Ultrastructural analysis was assessed as previously described, ${ }^{34}$ and observed using a Zeiss EM 902 transmission electron microscope (Zeiss, Oberkochem, Germany), at $90 \mathrm{kV}$, on ultra-thin sections $(50 \mathrm{~nm})$ stained with uranyl acetate and lead citrate.

Cell culture and flow cytometry analysis. RGC- 5 cells $^{35,36}$ were cultured in DMEM, $10 \% \mathrm{FBS}$ and $1 \%$ penicillin/streptomycin in a $5 \% \mathrm{CO}_{2}$ incubator. Cells were treated with $500 \mu \mathrm{M} \mathrm{PQ}$ (Sigma), $100 \mathrm{nM}$ rapamycin and $10 \mathrm{mM}$ 3-MA (Sigma) for 24 and $48 \mathrm{~h}$. When drugs were combined they were added simultaneously to the wells. After treatments cells were trypsinized and incubated with dichloro-fluorescein (DCF, $5 \mu \mathrm{M}$, Invitrogen) for $30 \mathrm{~min}$ at $37^{\circ} \mathrm{C}$ to measure intracellular ROS production in viable cells. $3,3^{\prime}$-dihexyloxacarbocyanine iodide $\left(\mathrm{DiOC}_{6}(3), 50 \mathrm{nM} 15 \mathrm{~min}\right.$; Invitrogen) was used to determine mitochondrial membrane potential, and propidium iodide ( $\mathrm{Pl}, 1 \mu \mathrm{g} / \mathrm{ml}$, Sigma) to determine cell viability. In total, 10000 cells were recorded in each analysis in an EPICS XL flow cytometer (Beckman Coulter, Barcelona, Spain).

Statistical analysis. Results are expressed as mean \pm S.E.M. of six in vitro experiments and at least five animals per group. Statistical analysis was performed using the JMP IN 4.0.3 software (JMP Software, Cary, NC, USA). For most analyses we used one-way ANOVAs including treatment as a fixed factor. If normality and homoscedasticity assumptions were not met we applied non-parametric tests. For all tests, the significance level was $P \leq 0.05$ (two-tailed) indicated by asterisk, and multiple testing was accounted for using Bonferroni correction. 


\section{Conflict of Interest}

The authors declare no conflict of interest.

Acknowledgements. We thank Noboru Mizushima for kindly supplying the GFP-LC3 and Atg5 ${ }^{\text {floxfllox }}$ mice and Carlos López-Otín for the Atg4B ${ }^{-/-}$mice. Professor Pedro de la Villa for electroretinogram determination and Dr. EJ de la Rosa, Dr. C López-Otín, Dr. T Suárez, Dr. P Vázquez, Dr. AM Cuervo and Dr. AM Tolkosvky for helpful comments on the manuscript and Owen Howard for English proofreading. This work has been supported by grants from the Fundación La Caixa and MICINN (SAF-2009-08086, SAF-2007-66175-C02-02 and RD07/ 0062/0008). NRM has a FPU fellowship from MICINN.

\section{Author Contributions}

NR-M performed all experiments, refined the methodology and performed ONTs in mice, experimental design, data analysis, and discussion and helped to write the manuscript. FG made the original observation of the presence of autophagosomes in axotomized rabbit retinas, developed the methodology for ONT in mice, DTMR experiments, inclusion for electron microscopy, intravitreal injections and critically read the manuscript. GM provided the Atg4 $4 B^{-1}$ mice and critically read the manuscript. PF participated in experimental design, data analysis and statistics and critically read the manuscript. PB directed the work, coordinated the general experimental design, data analysis and discussion and wrote the manuscript.

1. Isenmann S, Kretz A, Cellerino A. Molecular determinants of retinal ganglion cell development, survival, and regeneration. Prog Retin Eye Res 2003; 22: 483-543.

2. Villegas-Perez MP, Vidalsanz M, Rasminsky M, Bray GM, Aguayo AJ. Rapid and protracted phases of retinal ganglion-cell loss follow axotomy in the optic-nerve of adultrats. J Neurobiol 1993; 24: 23-36.

3. Tezel G. Oxidative stress in glaucomatous neurodegeneration: mechanisms and consequences. Prog Retin Eye Res 2006; 25: 490-513.

4. Yang Z, Klionsky DJ. Mammalian autophagy: core molecular machinery and signaling regulation. Curr Opin Cell Biol 2010; 22: 124-131.

5. Boya P, Gonzalez-Polo RA, Casares N, Perfettini J, Dessen P, Larochette N et al. Inhibition of macroautophagy triggers apoptosis. Mol Cell Biol 2005; 25: 1025-1040.

6. Ravikumar B, Sarkar S, Davies JE, Futter M, Garcia-Arencibia M, Green-Thompson ZW et al. Regulation of mammalian autophagy in physiology and pathophysiology. Physiol Rev 2010; 90: 1383-1435.

7. Nixon RA. Autophagy in neurodegenerative disease: friend, foe or turncoat? Trends Neurosci 2006; 29: 528-535.

8. Wong E, Cuervo AM. Autophagy gone awry in neurodegenerative diseases. Nat Neurosci 2010; 13: 805-811.

9. Hara T, Nakamura K, Matsui M, Yamamoto A, Nakahara Y, Suzuki-Migishima R et al. Suppression of basal autophagy in neural cells causes neurodegenerative disease in mice. Nature 2006; 441: 885-889.

10. Komatsu M, Waguri S, Chiba T, Murata S, Iwata J, Tanida I et al. Loss of autophagy in the central nervous system causes neurodegeneration in mice. Nature 2006; 441: 880-884.

11. Liang CC, Wang C, Peng X, Gan B, Guan JL. Neural-specific deletion of FIP200 leads to cerebellar degeneration caused by increased neuronal death and axon degeneration. $J$ Biol Chem 2010; 285: 3499-3509.

12. Pickford F, Masliah E, Britschgi M, Lucin K, Narasimhan R, Jaeger PA et al. The autophagy-related protein beclin 1 shows reduced expression in early Alzheime disease and regulates amyloid beta accumulation in mice. J Clin Invest 2008; 118: 2190-2199

13. Renna M, Jimenez-Sanchez M, Sarkar S, Rubinsztein DC. Chemical inducers of autophagy that enhance the clearance of mutant proteins in neurodegenerative diseases. J Biol Chem 2010; 285: 11061-11067.
14. Koike M, Shibata M, Tadakoshi M, Gotoh K, Komatsu M, Waguri S et al. Inhibition of autophagy prevents hippocampal pyramidal neuron death after hypoxic-ischemic injury. Am J Pathol 2008; 172: 454-469.

15. Ginet V, Puyal J, Clarke PG, Truttmann AC. Enhancement of autophagic flux after neonatal cerebral hypoxia-ischemia and its region-specific relationship to apoptotic mechanisms. Am J Pathol 2009; 175: 1962-1974.

16. Kim SH, Munemasa Y, Kwong JM, Ahn JH, Mareninov S, Gordon LK et al. Activation of autophagy in retinal ganglion cells. J Neurosci Res 2008; 86: 2943-2951.

17. Wang T, Lao U, Edgar BA. TOR-mediated autophagy regulates cell death in Drosophila neurodegenerative disease. J Cell Biol 2009; 186: 703-711.

18. Punzo $\mathrm{C}$, Kornacker $\mathrm{K}$, Cepko $\mathrm{CL}$. Stimulation of the insulin/mTOR pathway delays cone death in a mouse model of retinitis pigmentosa. Nat Neurosci 2009; 12: 44-52.

19. Knoferle J, Koch JC, Ostendorf T, Michel U, Planchamp V, Vutova P et al. Mechanisms of acute axonal degeneration in the optic nerve in vivo. Proc Natl Acad Sci USA 2010; 107: 6064-6069.

20. Park KK, Liu K, Hu Y, Smith PD, Wang C, Cai B et al. Promoting axon regeneration in the adult CNS by modulation of the PTEN/mTOR pathway. Science 2008; 322: 963-966.

21. Mizushima N, Yamamoto A, Matsui M, Yoshimori T, Ohsumi Y. In vivo analysis of autophagy in response to nutrient starvation using transgenic mice expressing a fluorescent autophagosome marker. Mol Biol Cell 2004; 15: 1101-1111.

22. Scherz-Shouval R, Elazar Z. Regulation of autophagy by ROS: physiology and pathology. Trends Biochem Sci 2011; 36: 30-38.

23. Jain A, Lamark T, Sjottem E, Larsen KB, Awuh JA, Overvatn A et al. p62/SQSTM1 is a target gene for transcription factor NRF2 and creates a positive feedback loop by inducing antioxidant response element-driven gene transcription. J Biol Chem 2010; 285: $22576-22591$.

24. Mariño G, Fernández AF, Cabrera S, Lundberg YW, Cabanillas R, Rodríguez $\mathrm{F}$ et al. Autophagy is essential for mouse sense of balance. J Clin Invest 2010; 120: 2331-2344.

25. Schlamp CL, Li Y, Dietz JA, Janssen KT, Nickells RW. Progressive ganglion cell loss and optic nerve degeneration in DBA/2J mice is variable and asymmetric. BMC Neurosci 2006; 7: 66.

26. McKinnon SJ, Schlamp CL, Nickells RW. Mouse models of retinal ganglion cell death and glaucoma. Exp Eye Res 2009; 88: 816-824.

27. Dehay B, Bové J, Rodríguez-Muela N, Perier C, Boya P, Vila M. Pathogenic lysosomal depletion in experimental Parkinson's disease: new therapeutic opportunities. J Neurosci 2010; 30: 12535-12544.

28. Ravikumar B, Vacher C, Berger Z, Davies JE, Luo S, Oroz LG et al. Inhibition of mTOR induces autophagy and reduces toxicity of polyglutamine expansions in fly and mouse models of Huntington disease. Nat Genet 2004; 36: 585-595.

29. Boland B, Kumar A, Lee S, Platt FM, Wegiel J, Yu WH et al. Autophagy induction and autophagosome clearance in neurons: relationship to autophagic pathology in Alzheimer's disease. J Neurosci 2008; 28: 6926-6937.

30. Carelli V, La Morgia C, Valentino ML, Barboni P, Ross-Cisneros FN, Sadun AA. Retinal ganglion cell neurodegeneration in mitochondrial inherited disorders. Biochim Biophys Acta 2009; 1787: 518-528.

31. Twig G, Elorza A, Molina AJ, Mohamed H, Wikstrom JD, Walzer G et al. Fission and selective fusion govern mitochondrial segregation and elimination by autophagy. EMBO $J$ 2008; 27: 433-446.

32. Ju WK, Kim KY, Lindsey JD, Angert M, Duong-Polk KX, Scott RT et al. Intraocular pressure elevation induces mitochondrial fission and triggers OPA1 release in glaucomatous optic nerve. Invest Ophthalmol Vis Sci 2008; 49: 4903-4911.

33. Baerga R, Zhang Y, Chen PH, Goldman S, Jin S. Targeted deletion of autophagy-related 5 (atg5) impairs adipogenesis in a cellular model and in mice. Autophagy 2009; 5: 1118-1130.

34. Mellén MA, de la Rosa EJ, Boya $P$. The autophagic machinery is necessary for removal of cell corpses from the developing retinal neuroepithelium. Cell Death Differ 2008; 15: $1279-1290$

35. Krishnamoorthy RR, Agarwal P, Prasanna G, Vopat K, Lambert W, Sheedlo HJ et al. Characterization of a transformed rat retinal ganglion cell line. Brain Res Mol Brain Res 2001; 86: 1-12.

36. Maffei L, Carmignoto G, Perry VH, Candeo P, Ferrari G. Schwann cells promote the survival of rat retinal ganglion cells after optic nerve section. Proc Natl Acad Sci USA 1990; 87: $1855-1859$.

\section{Supplementary Information accompanies the paper on Cell Death and Differentiation website (http://www.nature.com/cdd)}

